\title{
Percepção subjetiva do estresse em oficiais de arbitragem de basquetebol
}

\author{
Perceptionof stress in basketball officials
}

\author{
Mário Antônio de Moura Simim ${ }^{1}$ \\ Renato Melo Ferreira, ${ }^{2,}$ \\ Markus Vinicius Campos Souza ${ }^{3}$ \\ Alessandro Custódio Marques ${ }^{4}$ \\ Bruno Victor Correa da Silva ${ }^{5}$
}

\begin{abstract}
Resumo
Objetivo: analisar a percepção de estresse em árbitros após uma partida de basquete. Métodos: Participaram do estudo 20 árbitros de basquetebol (idade $=29,35 \pm 6,75$ anos; tempo de arbitragem $=$ $7,75 \pm 6,23$ anos), de nível municipal à internacional, credenciados e em atuação na Copa Revelar de Basquetebol. O instrumento utilizado foi o Teste de Estresse para Árbitros dos Jogos Esportivos Coletivos. Resultados: Os resultados indicam que os principais fatores causadores de estresse foram: Competição desorganizada $(2,75 \pm 1,52)$; Locais com falta de segurança $(2,35 \pm 0,88)$; Errar nos últimos minutos decisivos $(2,70 \pm 1,30)$; Errar em situações claras $(2,50 \pm 1,28)$; Errar seguidamente $(2,50 \pm 1,24)$; Atraso do transporte da organização $(2,40 \pm 1,14)$; Não receber o pagamento pelo jogo $(2,45 \pm 1,36)$; Chegar tarde ou atrasado no local do jogo $(2,55 \pm 1,36)$; Falta de responsabilidade do colega e outras pessoas $(2,45 \pm 1,43)$ e Não ter reconhecimento e/ ou valorização $(2,50 \pm 1,15)$. Conclusão: Concluímos que o estresse dos árbitros de basquete está relacionado a fatores sociais, relacionados com aorganização da competição e estrutura da modalidade e a fatores psicológicos, como errar na tomada de decisão de determinada situação.
\end{abstract}

Palavras-chave: estresse, árbitro, basquete.

\section{Abstract}

Objective: To analyze the perception of stress in officials after a basketball game. Method: 20 Basketball officials participated in the study (age $=29.35 \pm 6.75$ years, refereeing time $=7.75 \pm 6.23$ years), from municipal to international level, accredited and in action in the revealing Basketball Tournament. The instrument used was the Stress Test for Referees of Collective Sports Games. Results: The results indicate that the main stressors were: Disorganized events $(2.75 \pm 1.52)$; Places with poor safety $(2.35 \pm$ $0.88)$; Mistakes in the last decisive minutes $(2.70 \pm 1.30)$; Failures in clear situations $(2.50 \pm 1.28)$; Repetitive Mistakes ( $2.50 \pm 1.24)$; Coordinating transport delay $(2.40 \pm 1.14)$; Not receiving payment for the game $(2.45 \pm 1.36)$; Arriving late or late at the game site $(2.55 \pm 1.36)$; Lack of responsibility of colleague and others $(2.45 \pm 1.43)$ and No recognition and / or recovery $(2.50 \pm 1.15)$. Conclusion: We conclude that basketball official's post-game stress is caused by social factors related to the organization of the competition, the structure of the game, and to psychological factors, such as making mistakes in the decision making of a given situation.

Keywords: Stress, Official, Basketball.
Afiliação dos autores

${ }^{1}$ Grupo de pesquisas em Biodinâmica do Movimento Humano, Instituto de Educação Física e Esportes, Universidade Federal do Ceará, Fortaleza, Ceará, Brasil.

${ }^{2}$ Centro Desportivo da Universidade Federal de Ouro Preto, CEDUFOP, Universidade Federal de Ouro Preto, Ouro Preto, Minas Gerais, Brasil. ${ }^{3}$ Departamento de Ciências do Esporte, Universidade Federal do Triângulo Mineiro, Uberaba, Minas Gerais, Brasil. Mineiro, Uberaba, Minas Gerais, B
${ }^{4}$ Programa de Pós-graduação em Biodinâmica do Movimento e Esporte Universidade Estadual de Campinas, São Paulo, Brasil.

${ }^{5}$ Departamento de Ciências Biológicas, Ambientais e da Saúde, Centro Universitário de Belo Horizonte, Uni$\mathrm{BH}$, Belo Horizonte, Minas Gerais, Brasil.

\section{${ }^{*}$ Autor correspondente}

Centro Desportivo da Universidade Federal de Ouro Preto, CEDUFOP, Universidade Federal de Ouro Preto. Rua Dois, 110, Campus Universitário Ginásio de Esportes, CEP: 35400-000, Ouro Preto, Minas Gerais, Brasil. e-mail: renato.mf@hotmail.com

\section{Conflito de interesses}

Os autores declararam não haver conflito de interesses.

Processo de arbitragem

Recebido: 05/02/2018 Aprovado: 06/06/2018 


\section{Introdução}

Osjogos esportes coletivos são considerados modalidades esportivas complexas que envolvem processo dinâmico e contínuo de situações específicas, sob circunstâncias que exigem atenção, concentração e participação ativa ${ }^{1}$. No caso específico do basquetebol, novas regras surgem a fim de melhorar a dinâmica a partida ${ }^{2}$. Contudo essa evolução das regras nem sempre é acompanhada pelo árbitro, que deve tomar decisões em segundos'. Para tanto é imprescindível ao árbitro atenção constante durante o jogo além do controle emocional.

O árbitro pode ser considerado como umas das figuras mais importantes no ambiente esportivo uma vez que sua função se estabelece em um processo contínuo de tomada de decisões em situações com muita pressão ${ }^{1,2}$. Além de aplicar as regras da modalidade, ele ainda recebe pressão de outros personagens que estão envolvidos no contexto esportivo, tais como dirigentes, comissão técnica, torcida, mídia, entre outros ${ }^{4,5}$. As decisões dos oficiais de arbitragem acontecem por processos subjetivos de avaliação em situações de estresse2. Nesse sentido, ele está inserido em ambiente que envolve tomada de decisões em curtos intervalos de tempo ${ }^{6}$, exigindo eficiência e precisão ${ }^{7}$. Essas questões podem ser influenciadas pelo fenômeno do estresse, que pode iniciar-se a partir do momento que o árbitro recebe sua escalação de jogo (dias antes da partida) e nem sempre termina com o apito final ${ }^{5,8}$.

O conhecimento de situações de estresse específicas das partidas de basquetebol auxiliaria a preparação mental dos árbitros, tanto para os momentos que antecedem a competição, quanto para os momentos do jogo. É possível se preparar para a tomada de decisões diante das situações estudadas previamente, ou seja, perceber a situação antes que a mesma ocorra e então saber como agir, evitando maiores problemas e transtornos na partida. Assim, o objetivo do estudo foi analisar a percepção de estresse em oficiais de arbitragem após partida de basquetebol.

\section{Métodos}

Participaram do estudo 20 árbitros de basquetebol com idade de 29,35 $\pm 6,75$ anos e tempo de experiência de $7,75 \pm 6,23$ anos, de ambos os sexos (masculino: $n=16 ; 80 \%$ e feminino: $n$ $=4 ; 20 \%$ ), de vários níveis (municipal à internacional) credenciados e em atuação na Copa Revelar de Basquetebol, promovida pela Federação de Esportes Estudantis de Minas Gerais - FEEMG e Federação Mineira de Basquetebol - FMB.

$O$ instrumento utilizado foi o Teste de estresse para árbitros - TEPA6 composto de 69 questões agrupadas em três dimensões (biológica, social e psicológica) conforme apresentado na tabela 1. As questões são avaliadas por escala do tipo Likert onde: (0) Nada, (1) Pouquíssimo, (2) Pouco, (3) Muito, (4) Demais. No presente estudo, a confiabilidade interna foi testa para as dimensões (biológica $\alpha=0.80$; psicológica $\alpha=$ 0.93 e social $\alpha=0.95$ ) e instrumento ( $\alpha=0.97$ ), demonstrando alta confiabilidade conforme padronização estipulada na literatura9.

Tabela 1

Descrição das dimensões do instrumento

\begin{tabular}{|c|c|c|}
\hline Dimensão & Descrição & Itens \\
\hline Biológica & $\begin{array}{l}\text { Alterações, transformações e modificações do } \\
\text { estado fisiológico do árbitro (cansaço físico, sono, } \\
\text { fome e disfuncões orgânicas) }\end{array}$ & $08,09,10,39$ \\
\hline Psicológica & $\begin{array}{l}\text { Relações mentais de acordo com as situações } \\
\text { ocorridas dentro da competição } \\
\text { Inter-relações do árbitro com todas as pessoas } \\
\text { do ambiente que configuram sua ação e que podem } \\
\text { influenciar no seu rendimento esportivo. }\end{array}$ & $\begin{array}{r}19,20,23,24,25,26,28,29,30, \quad 32,46, \\
47,48,55,56,57,59,61,62,63,64,65,66,68 \\
01,02,03,04,05,06,07,11,12,13,14,15, \\
16,17,18,21,22,27,33,34,35,36,37,38,40,41 \\
42,43,44,45,49,50,51,52,53,54,58,60,67,69\end{array}$ \\
\hline
\end{tabular}

A pesquisa foi conduzida respeitando-se as normas éticas estabelecidas pelo Conselho Nacional de Saúde, sendo aprovada pelo Comitê de Ética local sob protocolo número 1.052.484 (COEP/UFTM). Os dados foram coletados após o termino da partida, sem pressão de tempo para que seja realizado o teste. Os árbitros participaram voluntariamente do estudo e previamente foram informados sobre os objetivos do estudo e assinaram o Termo de Consentimento Livre e Esclarecido.

Os dados são apresentados em média \pm desvio padrão, mediana e distribuição de frequência. Para a comparação entre as dimensões foi utilizado o Teste Friedman (X2). Em todas as comparações adotou-se o valor de $5 \%$ para identificar diferenças estatísticas. Adicionalmente, o tamanho do efeito (TE) foi utilizado de acordo com a seguinte escala de magnitude (Hopkins, 2010): 0 - 0,2 (trivial), > 0,2 (pequeno), > 0,6 (moderado), >1, 2 (grande), >2, 0 (muito grande). O teste Alpha crombach foi utilizado para analisar a confiabilidade interna das dimensões e do instrumento.

\section{Resultados}

Tabela 2

Resultados da percepção de estresse por situação.

\begin{tabular}{|c|c|c|c|c|}
\hline Itens & Dimensão & Média $\pm D P$ & Mediana & Escala \\
\hline Competição desorganizada & Social & $2,75 \pm 1,52$ & 4 & Demais \\
\hline Locais com falta de segurança & Social & $2,35 \pm 0,88$ & 3 & Muito \\
\hline Errar nos últimos minutos decisivos & Psicológico & $2,70 \pm 1,30$ & 3 & Muito \\
\hline Errar em situações claras & Psicológico & $2,50 \pm 1,28$ & 3 & Muito \\
\hline Errar seguidamente & Psicológico & $2,50 \pm 1,24$ & 3 & Muito \\
\hline Atraso do transporte da organização & Social & $2,40 \pm 1,14$ & 3 & Muito \\
\hline Não receber o pagamento pelo jogo & Social & $2,45 \pm 1,36$ & 3 & Muito \\
\hline Chegar tarde ou atrasado no local do jogo & Social & $2,55 \pm 1,36$ & 3 & Muito \\
\hline Falta de responsabilidade do colega e outras pessoas & Social & $2,45 \pm 1,43$ & 3 & Muito \\
\hline Não ter reconhecimento e/ ou valorização & Social & $2,50 \pm 1,15$ & 3 & Muito \\
\hline
\end{tabular}

A percepção de estresse é apresentada na figura 1 . O tamanho de efeito reportado entre as dimensões variou entre trivial $(T E=0,02)$ e pequeno $(T E=0,11)$. Não reportamos diferença entre as dimensões $(x 2=0,532 ; g l=2 ; p=0,767)$.

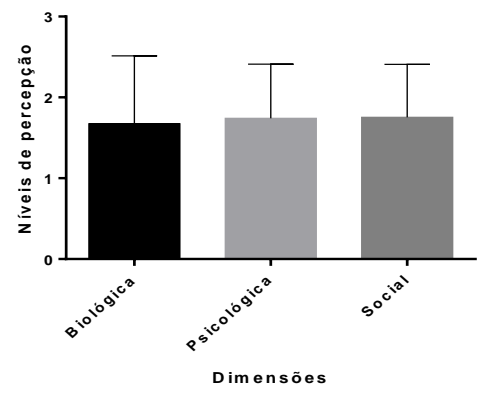

Figura 1. Nível de percepção entre a dimensões

A Tabela 2 apresenta os resultados da percepção das situações avaliadas como estresse elevado. 


\section{Discussão}

O presente estudo objetivou analisar a percepção de estresse em árbitros de basquetebol após uma partida, levando em consideração as situações que causam estresse. Os principais achados foram que fatores sociais e psicológicos se mostraram com níveis mais elevados de percepção de estresse.

Dentre as situações apontadas pelos árbitros a desorganização da competição se mostrou como fator de maior causa de estresse. Já há alguns anos o basquetebol no Brasil vem passando por constantes mudanças em sua organização de campeonatos, principalmente no que concerne as confederações e federações ${ }^{2}$. Nesse sentido, conflitos têm sido gerados no âmbito das organizações das competições, com mudanças no gerenciamento ${ }^{10}$. Exemplo recente desse fato foi a suspensão do Brasil de disputar qualquer competição internacional organizada pela Federação Internacional de Basquete (FIBA). A situação geradora da suspensão relaciona-se com o gerenciamento da modalidade pela Confederação Brasileira de Basquete (CBB), impactando no desempenho e insegurança dos árbitros em relação ao nível de organização das competições. Ademais destacamos que o árbitro deve ter condições adequadas de trabalho e caso isso não seja possível, esses fatores podem influenciar negativamente no desenvolvimento da competição. A organização da partida e competição envolve o local de jogo e consequentemente a segurança dos árbitros e de todos que compõem o cenário esportivo ${ }^{11}$.

Atraso no transporte para a competição também é um dos fatores que mais acarretam estresse nos árbitros investigados. Essa situação acarreta que o árbitro chegue tarde ou atrasado no local do jogo e está intimamente ligada às questões logísticas e organizacionais. Esse resultado é frequente em outros estudos reportados na literatura ${ }^{6,8,12}$, demonstrando necessidade de melhor organização por parte das instituições que regulam a modalidade no Brasil. Outra situação citada pelos árbitros foi a falta de segurança, situação constante e comum em várias modalidades ${ }^{13-16}$. Falta de segurança nos locais de competição implica em vulnerabilidade dos árbitros, sendo os mesmos susceptíveis à ameaças e tentativas de agressão por parte de atletas, treinadores e torcedores ${ }^{5}$.

Todas as situações citadas acima não são relacionadas ao desempenho do árbitro durante as partidas. Entretanto, essas situações organizacionais podem contribuir para rotatividade de árbitros e abandono da atividade de arbitragem ${ }^{17}$. Adicionalmente, os mesmos autores sugerem que experiências negativas no decorrer da arbitragem são responsáveis pelo abandono das funções de árbitro. Portanto, nós atribuímos os resultados mencionados acima à experiências negativas de atuação do árbitro no basquetebol.

É interessante chamar atenção para as questões relativas ao tempo de experiência e a falta de profissionalização do árbitro. Segundo Silva ${ }^{6}$ e Samulski e Silva ${ }^{11} 0$ árbitro é formado por sua experiência pratica em campo, com isso, o fato dos árbitros apresentarem tempo elevado de experiência e atuarem em diversos níveis e categorias (local à internacional), são fatores que podem contribuir para 0 controle do estresse na competição.

Árbitros de basquetebol geralmente atuam em equipe, fator que pode desencadear situações de estresse. Conforme apresentado no presente estudo, a "Falta de responsabilidade do colega e outras pessoas" foi indicada como situação causadora de estresse, principalmente pelo fato de trabalhar em grupo se tornar difícil, uma vez que a individualidade na forma de agir e pensar pode ser influenciar uma decisão durante a partida. Esse fato já havia sido reportado por Silva6 que destaca que a relação com o colega de arbitragem insere- se no ambiente social do jogo, sendo determinante também para o controle do estresse. Situações de preocupação com o desempenho pessoal e da equipe de arbitragem são as maiores causadoras de estresse para árbitros e oficiais de mesa do basquetebol ${ }^{5}$. Outros aspectos técnicos/pessoais que influenciam na atuação do árbitro foram os erros durante a partida, semelhante a outros trabalhos ${ }^{5,8,15}$. Cabe ressaltar que erros frequentes ou em situações claras podem mudar o resultado de uma partida e até mesmo de um campeonato, atribuindo mais responsabilidade para as questões sobre erros de arbitragem em momentos decisivos.

A principal limitação do estudo é o fato de a coleta de dados ser realizada em um único momento. Dessa maneira, a avaliação da percepção subjetiva de estresse dos árbitros pode ter sido influenciada por situações que ocorram no dia da coleta.

\section{Conclusão}

Conclui-se que as situações com maior percepção de estresse para os oficiais de basquete estão relacionadas a competição desorganizada, locais com falta de segurança, atraso no transporte, não receber o pagamento pelo jogo, falta de responsabilidade do colega e outras pessoas e não ter reconhecimento e/ ou valorização, além de erros no decorrer da partida.

\section{Referências}

1. Guillén F, Feltz DL. A conceptual model of referee efficacy. Front. Psychol. 2011. 2(18): 1-4.

2. Rodrigues HA, Leonardi TJ, Paes RR. Novas regras do basquetebol: estudo de caso sobre a percepcão de jogadores de uma equipe profissional. Conexões. 2013. 11(3):147-165.

3. Sousa MAM. Um olhar para os árbitros de futebol. Rev. bras. psicol. Esporte. 2016. 6(1):121-132.

4. Horn LGR, Reis LN. A profissionalização da arbitragem e sua influência na imagem dos árbitros: um estudo na ótica de profissionais ligados à gestão do futebol no Rio Grande do Sul. Revista Brasileira de Futsal e Futebol. 2016. 8(28):19-28.

5. Arns PC, Folle A, Leite RM. Nível de stress de oficiais de arbitragem do basquetebol catarinense. Cinergis. 2014; 15(1):05-09.

6. Silva SA. Construção e validação de um instrumento para medir o nível de estresse dos árbitros dos jogos coletivos. [Dissertação de Mestrado - Programa de Pós-Graduação em Ciências do Esporte]. Belo Horizonte (MG): Universidade Federal de Minas Gerais; 2004.

7. Silva AI, Rech CR. Somatotipo e composição corporal de árbitros árbitros assistentes da CBF. Rev. Bras. cineantropom. desempenho hum. 2008. 10(2):143-148.

8. Ferreira HC, Simim MA, Noce F, Samulski DM, Costa VT. Análise do estresse em árbitros de futsal. Coleç. Pesqui. Educ. Fís. 2009. 8(1):43 48.

9. Heo M, Kim N, Faith MS. Statistical power as a function of Cronbach alpha of instrument questionnaire items. BMC Med Res Methodol. 2015. 15(86): 1-9.

10. Capinussú JM, Lima WA. O retorno dos patrocinadores do Novo Basquete Brasil. RIGD. 2011; 1(2):122-131.

11. Samulski DM, Silva SA. Psicologia aplicada à arbitragem. In: Samulksi DM. Psicologia do Esporte: conceitos e novas perspectivas. Barueri: Manole, 2009. p 461-486.

12. De Rose Junior D, Pereira FP, Lemos RF. Situações específicas de jogo causadoras de "stress" em oficiais de basquetebol. Rev. Paul. Educ. Fís.2002. 16(2): 160-73.

13. De Rose Junior D. A competição como fonte de estresse no esporte. Rev. Bras. Ciên. E Mov. 2002. 10(4):19-26.

14. Dorcsh KD, Paskevich DM. Stressful experiences among six certification levels of ice hockey officials. Psychol Sport Exerc. 2007. 8(4): 585-593.

15. Gabardo AL, Comparim MSCV. Fatores causadores de stress em equipe de arbitragem da Federação Paranaense de Basketball. Revista Eletrônica de Educação Física. 2008.

16. Costa VT, Ferreira RM, Penna EM, Costa IT, Noce F, Simim MAM Análise estresse psíquico em árbitros de futebol. Rev. bras. psicol. esporte. 2010. 3(2):02-16.

17. Warner S, Tingle JK, Keller P. Officiating Attrition: The Experiences of Former Referees Via a Sport Development Lens. Journalof Sport Management. 2013. 27(4):316-328. 